To the Editors:

\title{
Perception of pain during episiotomy in a tertiary care centre in Sri Lanka
}

\author{
Ceylon Medical Journal 2011; 56: 41-42
}

Episiotomy is used almost routinely in vaginal deliveries in Sri Lanka and the norm is for it to be performed without anaesthesia. This is due to the popular belief that episiotomy could be performed painlessly without anaesthesia if it is performed at the peak of a contraction when the perineum is at full stretch. This practice is also convenient, since nurses and midwives involved in normal deliveries in Sri Lanka are not licensed to administer a local anaesthetic.

An analytical cross sectional study was carried out to assess pain felt at the time of episiotomy. Fifty primigravidae who had a normal delivery without epidural analgesia were recruited from wards where a local anaesthetic is administered at the time of performing episiotomy and fifty from where it is not. A systematic sampling method was used. Data was gathered using an interviewer administered questionnaire between 6-12 hours postpartum. Degree of pain during episiotomy was measured by both mothers and observers using visual analogue scale (VAS), which was calibrated from zero to ten.

The VASs were compared using the Mann-Whitney $\mathrm{U}$ test. To ensure that the mother's scoring on the VAS was a reflection of perception of pain, three of the authors (RA, MA and TA) acted as observers for 10 (20\%) deliveries of each arm. Agreement between VAS scores between observers and mothers were tested using the Kappa test. Table 1 shows the VAS scoring system used and the scores given by mothers in relation to pain felt while performing the episiotomy. The measure of agreement between the values regarding perception of pain between mothers and observers was 0.908 and 0.863 (K>0.80) respectively, confirming that mothers had indicated the pain of episiotomy without confusing it with labour pain.

The median VAS score for pain during episiotomy in mothers who were given local anaesthesia was $0.00(\mathrm{IQR}=$ $0.00)$ and in mothers who were not, this was $7(\mathrm{IQR}=3)$. The median VAS score for pain was significantly lower in the mothers who were given local anaesthesia than in those who were not $(p=0.0001)$. The pain during administration of the local anaesthetic was assessed separately. Twenty one mothers (42\%) indicated zero and 28 (56\%) one to four in VAS. Maximum value indicated was 7 , by a single mother. All mothers (100\%) who received local anaesthesia and 45 (90\%) mothers who did not receive it said they would recommend administering local analgesia prior to episiotomy.

This study shows that the belief that episiotomy does not require anaesthesia if it is performed at the 'correct' time is baseless. Women experience considerable pain during episiotomy, which can be prevented by using local anaesthetics.

Table 1. VAS scoring system and the scores given by women regarding pain relating to performing the episiotomy

\begin{tabular}{lcccc}
\hline & $\begin{array}{c}\text { With use of } \\
\text { local anaesthetic } \\
\mathrm{n}=50\end{array}$ & $\%$ & $\begin{array}{c}\text { Without use of } \\
\text { local anaesthetic } \\
\mathrm{n}=50\end{array}$ & $\%$ \\
\hline Median VAS score (IQR) & 0 & & 7 & $p=0.0001$ \\
Zero (No pain was felt) & 41 & 82.0 & 4 & 8.0 \\
1-4 (Mild pain) & 7 & 14.0 & 1 & 2.0 \\
5 (Moderate pain) & 1 & 2.0 & 9 & 18.0 \\
6-9 (Severe but bearable pain) & 1 & 2.0 & 27 & 54.0 \\
10 (Excruciating) & 0 & 0.0 & 9 & 18.0 \\
\hline
\end{tabular}




\section{Acknowledgements}

We wish to acknowledge cooperation of the staff of wards 3, 5, 15 and 16 of the De Soysa Hospital for Women and the Department of Community Medicine of the Faculty of Medicine, Colombo and Dr. Charukshi Arambepola for the help and advice given during all stages of this student research project.

\section{H Senanayake ${ }^{1}$, R Adikaram ${ }^{1}$, T Alwis $^{1}$, M Ahmad $^{1}$, B B M J P Gunathilaka ${ }^{1}$}

${ }^{1}$ Department of Gynaecology and Obstetrics, Faculty of Medicine, University of Colombo, Sri Lanka.

Correspondence: HS, e-mail <senanayakeh@gmail.com>. Received 23 March and revised version accepted 23 September 2010. Competing interests: none declared. 